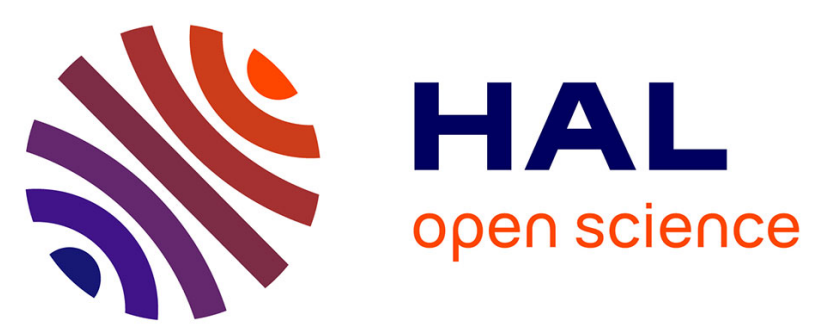

\title{
A hybrid framework for single tree detection from airborne laser scanning data: A case study in temperate mature coniferous forests in Ontario, Canada
}

Junjie Zhang, Gunho Sohn, Mathieu Brédif

\section{- To cite this version:}

Junjie Zhang, Gunho Sohn, Mathieu Brédif. A hybrid framework for single tree detection from airborne laser scanning data: A case study in temperate mature coniferous forests in Ontario, Canada. ISPRS Journal of Photogrammetry and Remote Sensing, 2014, 98, pp.44-57. 10.1016/j.isprsjprs.2014.08.007 . hal-01883864

\section{HAL Id: hal-01883864 \\ https://hal.science/hal-01883864}

Submitted on 28 Sep 2018

HAL is a multi-disciplinary open access archive for the deposit and dissemination of scientific research documents, whether they are published or not. The documents may come from teaching and research institutions in France or abroad, or from public or private research centers.
L'archive ouverte pluridisciplinaire $\mathbf{H A L}$, est destinée au dépôt et à la diffusion de documents scientifiques de niveau recherche, publiés ou non, émanant des établissements d'enseignement et de recherche français ou étrangers, des laboratoires publics ou privés. 
This manuscript was accepted for the publication by ISPRS Journal on August 15, 2014. DOI: 10.1016/j.isprsjprs.2014.08.007

Title:

2 A Hybrid Framework for Single Tree Detection from Airborne Laser Scanning Data: A Case Study in

3 Temperate Mature Coniferous Forests in Ontario, Canada

5 Authors:

6 Junjie Zhang (1), Gunho Sohn *(1), Mathieu Brédif (2)

\section{Affiliations:}

9

10

(1) GeoICT lab, Department of Earth and Space Science \& Engineering, York University, 4700 Keele Street, Toronto, ON M3J 1P3, Canada

11

(2) Université Paris Est, IGN, MATIS 73, avenue de Paris, 94165 Saint-Mandé, France

12

* Corresponding author.

13

14 Contact information:

15 JZ: +1 4167362100 ext. 22611; junjiez@yorku.ca

16 GS: +1 416650 8011; gsohn@yorku.ca

17

MB: +33 1439883 19; mathieu.bredif@ign.fr

18 
This manuscript was accepted for the publication by ISPRS Journal on August 15, 2014. DOI: 10.1016/j.isprsjprs.2014.08.007

\section{Abstract}

This study presents a hybrid framework for single tree detection from airborne laser scanning (ALS) data by integrating low-level image processing techniques into a high-level probabilistic framework. The proposed approach modelled tree crowns in a forest plot as a configuration of circular objects. We took advantage of low-level image processing techniques to generate candidate configurations from the canopy height model (CHM): the treetop positions were sampled within the over-extracted local maxima via local maxima filtering, and the crown sizes were derived from marker-controlled watershed segmentation using corresponding treetops as markers. The configuration containing the best possible set of detected tree objects was estimated by a global optimization solver. To achieve this, we introduced a Gibbs energy, which contains a data term that judges the fitness of the objects with respect to the data, and a prior term that prevents severe overlapping between tree crowns on the configuration space. The energy was then embedded into a Markov Chain Monte Carlo (MCMC) dynamics coupled with a simulated annealing to find its global minimum. In this research, we also proposed a Monte Carlo-based sampling method for parameter estimation. We tested the method on a temperate mature coniferous forest in Ontario, Canada and also on simulated coniferous forest plots with different degrees of crown overlap. The experimental results showed the effectiveness of our proposed method, which was capable of reducing the commission errors produced by local maxima filtering, thus increasing the overall detection accuracy by approximately $10 \%$ on all of the datasets.

\section{Keywords:}

LiDAR, Forestry, Single Tree Detection, Local Maxima Filtering, Marker-controlled Watershed Segmentation, Stochastic Model, Energy Minimization, MCMC 
This manuscript was accepted for the publication by ISPRS Journal on August 15, 2014. DOI: 10.1016/j.isprsjprs.2014.08.007

\section{Introduction}

41 Remote sensing techniques have become an integral part of forest inventory to provide accurate, precise

42 and timely forest and tree characteristics at different scales to support practices of forest management and

43 planning (Dubayah and Drake, 2000; Naesset et al., 2004; Tomppo et al., 2002; Wulder, 1998; Xie et al.,

44 2008). Among these techniques, small-footprint airborne laser scanning (ALS), also known as airborne

45 LiDAR, has rapidly gained popularity in forest inventory in recent decades. The unique capability of ALS

46 to directly measure the 3D structural information of trees and the elevation of the terrestrial surface under

47 the canopy in forests makes ALS an alternative to traditional passive optical remote sensing technology,

48 or even the preferred method, to derive certain forest parameters, such as canopy height, crown

49 dimensions, stand volume, basal area, and above-ground biomass (Bortolot and Wynne, 2005; Hyyppä

50 and Inkinen, 1999; Means et al., 2000; Næsset, 1997; Naesset, 2002).

51 Characterization of forest resources using ALS can be broadly categorized into area-based approaches

52 (ABAs) and individual-tree-based approaches (ITDs) (Hyyppä et al., 2008). ABAs rely on the statistical

53 principle and predicts forest attributes based on parametric regression or nonparametric imputation

54 models built between field measured variables and features derived from ALS data (Maltamo et al., 2006;

55 Naesset, 2002). ABAs can perform under a low ALS point density, and is the method currently applied in

56 operational forest inventory to provide a wall-to-wall estimation of forest attributes (Naesset, 2004; White

57 et al., 2013). ITDs measure or predict tree-level variables on the basic unit of the individual trees from

58 ALS data and then aggregate them to obtain stand-level forest inventory results (Hyyppä et al., 2012).

59 Despite the added costs and amount of information to store and process high-density ALS data, ITDs are

60 of significant interest in forest inventory and is a motivating research topic. The primary advantage of

61 ITDs over ABAs is the supply of tree lists and the ability to directly derive the true stem distribution

62 series, which would result in better prediction for timber assortments (Vastaranta et al., 2011a).

63 Generally, this information is invaluable in forest planning-related simulation and optimization, logging 
This manuscript was accepted for the publication by ISPRS Journal on August 15, 2014. DOI: 10.1016/j.isprsjprs.2014.08.007

64 operation planning and wood supply logistics (Vastaranta et al., 2011b), e.g., detection of harvest trees and forest growth determination (Yu et al., 2004). Another advantage of ITDs is that they can reduce the amount of or potentially replace the expensive fieldwork required for ABAs (Hyyppä et al., 2008; Vastaranta et al., 2012). Additionally, tree species classification based on ITD has been reported in recent studies (Brandtberg, 2007; Heinzel and Koch, 2011; Orka et al., 2009; Suratno et al., 2009), which could potentially improve the prediction of species-specific forest attributes (Heurich, 2008; Yao et al., 2012; Yu et al., 2010). Furthermore, the combination of ITD and ABA, called the semi-ITD method, to improve the estimation accuracy has also been viewed as a future method for forest inventory (Breidenbach et al.,

72 2010; Hyyppä et al., 2012; Vastaranta et al., 2012). Therefore, individual tree detection techniques are

73 still of significant importance from the practical forestry viewpoint.

74 Accordingly, numerous methods have been proposed to detect single trees from ALS data. Most of the methods focus on the generation of the canopy height model (CHM), which provides an accurate representation of the outer surface of the tree canopy. The peaks and valleys on the CHM generated from high-density ALS data are better estimations of treetop positions and crown edges than can be obtained from aerial photographs or satellite imageries. Therefore, many studies have extended methods developed for passive optical imageries to detect single trees from ALS data. Those methods include, but are not limited to, local maxima filtering (Popescu et al., 2002; Wulder et al., 2000), region growing (Erikson, 2003; Solberg et al., 2006), valley following (Gougeon, 1995; Leckie et al., 2003), template matching (Korpela et al., 2007; Pollock, 1996), watershed segmentation and its variance marker-controlled

83 watershed segmentation (Chen et al., 2006; Pyysalo and Hyyppa, 2002; Wang et al., 2004), and multi84 scale segmentation (Brandtberg and Walter, 1998; Brandtberg et al., 2003). 
This manuscript was accepted for the publication by ISPRS Journal on August 15, 2014. DOI: 10.1016/j.isprsjprs.2014.08.007

filtering on the CHMs, attempting to overcome errors of omission and commission associated with fixed window local maxima filtering (Hyyppä et al., 2001).

91 Once the treetops are detected, MCWS is well suited to delineate the tree crown segments from the CHM.

92 MCWS, which possesses the advantages of other segmentation methods of region growing and edge

93 detection, was introduced by Meyer and Beucher (1990) to overcome the over-segmentation problem of

94 ordinary watershed segmentation. In MCWS, user-specified markers are used as the marker function to

95 perform the segmentation; for additional details, see Gonzalez and Woods (2008). In the resultant

96 segmentation, there will be one segment corresponding to each marker; in the case of single tree

97 detection, one tree crown will be captured by one treetop. This result indicates the detection accuracy of

98 MCWS, subject to the accuracy of the pre-determined local maxima as true treetops in the previous stage.

99 The issue with LM is the selection of the filter window size and the determination of the relationship

100 between the crown size and the tree height. In the comparison of tree detection algorithms (Kaartinen et

101 al., 2012), the local maxima-based approach tends to produce high commission errors. Especially in

102 coniferous forests, spurious treetops are detected within the tree crowns from large branches. In other

103 cases, local maxima filtering produces a low commission error, and the omission error often increases

104 because small tree crowns are more likely to be undetected (Gebreslasie et al., 2011).

105 Probabilistic methods represent another branch of powerful tools in image analysis. These methods have

106 proven to hold great promise in solving inverse problems, including image segmentation, image

107 restoration, and feature extraction (Descombes and Zerubia, 2002). In particular, stochastic models have

108 evolved from random fields to object processes, and the work has shifted from an early focus on 'low-

109 level' tasks that aim to de-noise, sharpen, and segment images to solving 'high-level' tasks of feature

110 recognition, i.e., describing an image by its content (Van Lieshout, 2009). Additional details on low-level

111 and high-level image analysis tasks can be found in Sonka et al. (2008). 
This manuscript was accepted for the publication by ISPRS Journal on August 15, 2014. DOI: 10.1016/j.isprsjprs.2014.08.007

112 Marked point processes, detailed in Van Lieshout (2000), are among the most efficient stochastic models

113 used to exploit the random variables whose realizations are configurations of geometric objects or shapes.

114 Generally, in these processes, after a probability distribution measuring the quality of each object

115 configuration is defined in the configuration space, the maxima density estimator is searched for by the

116 Markov Chain Monte Carlo (MCMC) sampler (Hastings, 1970) coupled with conventional simulated

117 annealing (Metropolis et al., 1953). This process has led to convincing experimental results in various

118 image analysis and feature extraction applications, such as road networks extraction (Lacoste et al., 2005),

119 road mark detection (Tournaire and Paparoditis, 2009), and 3D building reconstruction (Lafarge et al.,

120 2008; Ortner et al., 2008; Tournaire et al., 2010).

121 Likewise, several stochastic models have been proposed to detect tree crowns from remote sensing data.

122 Descombes and Pechersky (2006) have presented a three-state Markov Random Field (MRF) model to

123 detect the tree crowns from aerial imageries. This approach addressed the problem as an image

124 segmentation problem and works on the pixel level. Each pixel is assigned to one of the following three

125 states: (i) vegetation, (ii) background, and (iii) center of trees. Although the MRF was defined on the

126 pixel level, the label update was performed on the object level using elliptical templates of crowns.

127 Furthermore, Perrin $(2005,2006)$ has employed marked point processes to detect tree crowns in

128 plantations from color infrared (CIR) aerial imageries. Tree crowns in the remote sensing image are

129 modeled as a configuration of discs or ellipses. In both of the studies, tree crowns were detected by

130 maximizing a Bayesian criterion, such as Maximum A Posteriori (MAP), which became an energy

131 minimization problem and was solved in a simulated annealing framework.

132 These stochastic models provide a powerful framework to allow the inclusion of spatial interactions

133 between objects in the prior while enabling a measure of consistency between objects and the image in

134 the data term. However, the inherited property of stochastic models requires exploration of a large

135 configuration space searching for the optimal configuration, especially for non-data-driven models, which 
This manuscript was accepted for the publication by ISPRS Journal on August 15, 2014. DOI: 10.1016/j.isprsjprs.2014.08.007

136 do not employ any low-level information that can be extracted from the images. The optimization process

137 is typically lengthy and computationally expensive.

138 This study presents a hybrid framework used to detect single trees from ALS data by integrating the low-

139 level image processing techniques, i.e., LM and MCWS, into a high-level probabilistic model. The

140 proposed model aims to improve the detection accuracy compared with traditional LM. Moreover, this

141 model samples in a reduced configuration space by utilizing image features extracted by LM and MCWS,

142 which potentially accelerate the optimization process compared with classical stochastic models, e.g.,

143 marked point processes. The estimation of parameters is another issue. In most cases, the parameters are

144 tuned by trial and error. We address the problem of parameter estimation by proposing a Monte Carlo

145 based method.

146 This paper is organized as follows. Section 2 describes the study area and the data used in the study.

147 Section 3 is dedicated to the formulation of our proposed model. We provide an overview of the general

148 framework of energy modeling for the stochastic model, followed by detailing the model design from the

149 configuration space definition to the energy formulation, parameter estimation and model optimization.

150 Finally, an accuracy assessment method is included. The experimental results of the parameter estimation

151 and tree detection on real and simulated ALS data are given in Section 4, and Section 5 presents a

152 discussion on the proposed model and the achieved results. Conclusions and certain perspectives for

153 future studies are outlined in Section 6.

\section{Materials}

\section{$155 \quad 2.1 \quad$ Study Area}

156 The study area is a temperate mature coniferous forest located in the Great Lakes-St. Lawrence region

157 approximately $60 \mathrm{~km}$ east of Sault Ste. Marie, Ontario, Canada (Figure 1(a)). The natural vegetation

158 dominant in the coniferous forest is eastern white pine (Pinus strobus) and jack pine (Pinus banksiana),

159 mixed with some red pine (Pinus resinosa) and black spruce (Picea mariana). The forest has an 
This manuscript was accepted for the publication by ISPRS Journal on August 15, 2014. DOI: 10.1016/j.isprsjprs.2014.08.007

160 intermediate dense canopy with some open space. The canopy height is homogenous with an average

161 height of approximately $20 \mathrm{~m}$. There are some small white pines and shrubs growing in the understory

162 with a height of approximately 2-3 m (Figure 1(b)-(c)).

$163 * * * * *$ Approximate position of Figure $1 * * * * *$

\section{$164 \quad 2.2 \quad$ Field Survey}

165 To test the proposed single tree detection model, three plots with sizes of $82 \times 95 \mathrm{~m}, 50 \times 50 \mathrm{~m}$ and $80 \times$

$16680 \mathrm{~m}$ were selected, and a field survey was conducted in August 2009. The forest mensuration campaign

167 determined the tree height $\left(h_{i}, \mathrm{~m}\right)$ with a Vertex hypsometer and the diameter at beast height (DBH) with

168 a DBH tape. The positions of trees with a height greater than $5 \mathrm{~m}\left(h_{i} \geq 5\right)$ were determined using GPS

169 and the total station. The crown width and species were also measured and recorded. The stem densities

170 of trees with a value of $h_{i} \geq 5$ are 154/ha, 160/ha and 190/ha, with increasing values for the three study

171 plots.

\section{$172 \quad 2.3 \quad$ Airborne Laser Scanning Data}

173 The ALS data were acquired over the study area by a Riegl LMS-Q560 laser scanner during the same

174 period as the field work. The flight was performed at a height of approximately $300 \mathrm{~m}$ above the ground

175 with a maximum scanning angle of $22.5^{\circ}$, rendering a swath width of approximately $300 \mathrm{~m}$. The flight

176 line was designed to pass over the planned forest plots; therefore, they were located in the middle part of

177 the swath, and the obscure effect of the crowns can be minimized for the plots of interest. The device

178 recorded full-waveforms that were processed into discrete point clouds with up to 5 returns per pulse. The

179 data collection configuration yielded a high point density of approximately 30 points per $\mathrm{m}^{2}$ over the

180 forested area. The returns were classified as ground and vegetation points using TerraScan software

181 (TerraSolid Ltd, Helsinki, Finland). The CHM with a resolution of $0.5 \mathrm{~m}$ was derived as the difference

182 between the digital surface model (DSM) and the digital elevation model (DEM), interpolated from

183 vegetation points and ground points, respectively (Hyyppä et al., 2001). 
This manuscript was accepted for the publication by ISPRS Journal on August 15, 2014. DOI: 10.1016/j.isprsjprs.2014.08.007

184

185

186

187

188

189

190

191

192

193

194

195

196

197

198

199

200

201

\subsection{Simulated ALS Data}

Vauhkonen et al. (2012) noted that the performance of the ITD algorithms typically depends on the tree density and the spatial distribution of trees, i.e., clustering patterns. To test the robustness of the proposed model more thoroughly, simulated ALS data of coniferous forest plots with a higher stem density than real forest plots and different degrees of crown overlap were also prepared in our study. First, three forest plots, each with a size of $100 \times 100 \mathrm{~m}$, were generated with a hard-core process in which the crown overlap was controlled by the interaction distance specified in the hard-core process. The smaller the interaction distance in the hard-core process, the more likely the tree objects will be overlapped in the resultant plots. Figure 2(a)-(c) show the three resulting point processes. With an increasing degree of crown overlap, the tree density in the plots also increases. The stem densities of trees with a value of $h_{i} \geq$ 5 in the three forest plots are 186/ha, 234/ha and 261/ha, respectively.

*****Approximate position of Figure $2 * * * * *$

ALS point clouds of individual trees were then selected according to the crown size from a coniferous tree template library and placed in each position to synthesize the ALS data of the forest plot. The tree template library was prepared from ALS data acquired from the study area we surveyed. A more detailed procedure can be found in Zhang and Sohn (2010). The generated ALS point clouds viewed from the nadir direction are shown in Figure 2(d)-(f). The plots from left to right show forest plots with separated, touching and overlapping tree crowns, respectively.

In the simulated forest plots, the tree position, height and crown size are exactly known, therefore providing ideal reference data to examine the performance of our proposed model under different forest conditions. The simulated ALS data can also be used to validate the parameter estimation method proposed in Section 3.5 and to investigate the influence that the degree of crown overlap has on the parameter setting in the proposed model. 
This manuscript was accepted for the publication by ISPRS Journal on August 15, 2014. DOI: 10.1016/j.isprsjprs.2014.08.007

\section{Methodology}

\section{$208 \quad 3.1$ General Framework of Energy Modeling for the Stochastic Models}

209 In a probabilistic framework, feature extraction or object detection from remotely sensed data can be

210 viewed as an inverse problem. In object oriented stochastic models, features or objects are represented as

211 a configuration of geometric shapes or objects. To find the best configuration $\mathbf{x}$ based on the observed

212 data $\mathbf{y}$ (the image), we must find the configuration $\hat{\mathbf{x}}$ maximizing the posterior probability, according to

213 the following equation:

$$
\hat{\mathbf{x}}=\arg \max _{\mathbf{x} \in \Omega} \mathbb{P}(X=\mathbf{x} \mid Y=\mathbf{y})
$$

214 where $\Omega$ is the configuration space in which $\mathbf{x}$ resides. $X$ and $Y$ are two random variables.

215 The probability of the model can also be specified in the form of a Gibbs energy $U(\mathbf{x})$, which implicitly

216 depends on the constant value $\mathbf{y}$ given by the observation:

$$
\mathbb{P}(X=\mathbf{x} \mid Y=\mathbf{y})=\frac{1}{Z} e^{-U(\mathbf{x})}
$$

217 where $Z$ is a normalizing constant such that $Z=\int_{\mathbf{x} \in \Omega} e^{-U(\mathbf{x})}$. The issue is then reduced to the energy

218 minimization problem of finding the Maximum A Posteriori estimator $\hat{\mathbf{x}}=\arg \max _{\mathbf{x} \in \Omega} \mathbb{P}(X=\mathbf{x} \mid Y=\mathbf{y})$,

219 which is equivalent to finding the configuration minimizing the Gibbs energy $U($.$) , i.e., \hat{\mathbf{x}}=$

$220 \arg \min _{\mathbf{x} \in \Omega} U(\mathbf{x})$. Generally, an MCMC embedded simulated annealing is used to find the optimal

221 configuration $\hat{\mathbf{x}}$. The optimization process is particularly interesting because the complex computation of

222 the normalizing constant $Z$ is avoided.

\section{$223 \quad 3.2$ Overall Workflow of the Proposed Model}

224 The flow chart of the proposed method is shown in Figure 3. As our primary contribution, the blue blocks 225 show the process how we construct a constrained configuration space for tree detection, by taking 
This manuscript was accepted for the publication by ISPRS Journal on August 15, 2014. DOI: 10.1016/j.isprsjprs.2014.08.007

226 advantages of low-level image processing techniques, which is detailed in Section 3.3. The red block

227 involves techniques of energy formulation and parameter estimation, which are covered in Section 3.4

228 and Section 3.5, respectively. The optimization process illustrated by the yellow blocks is described in

229 Section 3.6.

$230 * * * * *$ Approximate position of Figure $3 * * * * *$

\section{$231 \quad 3.3 \quad$ Configuration Space Definition of the Proposed Model}

232 Let us first recall the configuration space definition in the marked point process. In remote sensing 233 images, the distribution of tree crowns in forests can be represented by a marked point process of disks.

234 The associated space $\mathcal{S}$ can be written according to the following equation:

$$
\mathcal{S}=\mathcal{P} \times \mathcal{M}=\left[0, X_{M}\right] \times\left[0, Y_{M}\right] \times\left[r_{m}, r_{M}\right]
$$

235 where $X_{M}$ and $Y_{M}$ are the width and height of the image $\mathcal{J}$, respectively, and $\left(r_{m}, r_{M}\right)$ are the minimum

236 and maximum radii of the disks in the configuration, respectively. Note that $x=(p, r) \in \mathcal{S}$ is a tree

237 object, where $p \in \mathcal{P}$ is its position and $r \in \mathcal{M}$ its radius. The configuration space $\Omega$ of the marked point

238 process of the tree crowns can be written according to the following equation:

$$
\Omega=\bigcup_{n=0}^{\infty} \Omega_{n}, \Omega_{n}=\left\{\left\{x_{1}, \ldots, x_{n}\right\} \subset \mathcal{S}\right\}
$$

239 that contains all of the configurations of a finite number of tree objects $x_{i}$ of $\mathcal{S}$.

240 In this study, we seek to construct a constrained configuration space $\Omega_{T} \subset \Omega$ in which the optimal or near

241 optimal configuration resides. We will then limit the search for the optimal configuration in the

242 constrained space $\Omega_{T}$, which could significantly reduce the computation demand of random sampling in $\Omega$

243 in the optimization process. 
This manuscript was accepted for the publication by ISPRS Journal on August 15, 2014. DOI: 10.1016/j.isprsjprs.2014.08.007

244 We begin by constructing a CHM image, representing the height of the tree crowns above ground from 245 the classified ALS data. Then, we extract the local maxima as potential treetops from the CHM using 246 local maxima filtering with a variable window size method adapted from Popescu et al. (2002). Our rule

247 is to detect as many true treetops and reduce omission errors in the first stage. Therefore, the filters of the

248 LM are set with relative small size empirically based on the priori knowledge about the plots to over-

249 populate initial 'treetops'. Let $T$ represents the set of extracted local maxima: $T=\left\{t_{1}, \ldots, t_{N}\right\}, \forall i \in$

$250\{1, \ldots, N\}, t_{i} \in \mathcal{P}$, where $N$ is the total number of local maxima extracted. The true treetops within the set

251 of local maxima $T$ are noted as $T^{o} \subset T$.

252 Given any subset of local maxima $C \subset T$, they can be used as markers in marker-controlled watershed 253 segmentation to obtain a partition $S(C)=\left\{s_{C_{1}}, \ldots, s_{C_{n(C)}}\right\}$ of the CHM, where $s_{C_{i}}$ is the corresponding 254 segment of the local maxima $t_{C_{i}} \in C . S(C)$ is a low-level presentation of the CHM image, and the set of 255 segments are assumed to be a reasonable approximation of the tree crowns with respect to the set of local 256 maxima $C$, where $n(c)$ is the number of local maxima in $C$.

257 A tree object $x_{C_{i}}=\left(t_{C_{i}}, r_{C_{i}}\right)$ is then defined by its location and radius on the segment $s_{C_{i}}$, where the tree 258 location is the corresponding local maximum $t_{C_{i}}$, and the radius $r_{C_{i}}$ is calculated as the average radius of 259 the segment $s_{C_{i}}$. A configuration $\mathbf{x}(C)=\left\{x_{C_{1}}, \ldots, x_{C_{n(C)}}\right\}$ is then constructed from the set of local maxima $260 C$. The entire procedure of configuration construction is illustrated in Figure 4. *****Approximate position of Figure $4 * * * * *$

262 We note all of the configurations generated from the subsets of local maxima $T$ as $\Omega_{T}=\{\mathbf{x}(C), C \subset T\}$.

263 Apparently, $\Omega_{T}$ is a discrete subspace of the configuration space $\Omega$, which cardinality is

$264 \operatorname{card}(\{\mathbf{x}(C), C \subset T\})=\operatorname{card}(\{C, C \subset T\})=2^{\operatorname{card}(T)}$. In this manner, we build a constrained

265 configuration space $\Omega_{T}$ from which to sample the optimal configuration. 
This manuscript was accepted for the publication by ISPRS Journal on August 15, 2014. DOI: 10.1016/j.isprsjprs.2014.08.007

\subsection{Energy Formulation}

267 As previously mentioned, the Gibbs energy $U(\mathbf{x})$ is defined on the configuration space to measure the 268 goodness or cost of each object configuration. The Gibbs energy can be further expressed as a weighted 269 sum of a prior term $U_{p}(\mathbf{x})$ that favors a specific spatial pattern in configuration $\mathbf{x}$ and a data term $U_{d}(\mathbf{x})$

270 that quantifies the quality of the configuration with respect to the data, according to the following 271 equation:

$$
U(\mathbf{x})=\alpha U_{d}(\mathbf{x})+(1-\alpha) U_{p}(\mathbf{x})
$$

272 where $\alpha \in[0,1]$ specifies the relative weights of the two energy terms.

273 We intend to make simple and effective choices for the design of each energy term. The basic

274 assumptions are the geometric properties of trees in mature coniferous forests in which treetops are

275 typically located in the central part of tree crowns, and tree crowns are of a circular shape when viewed

276 from the nadir direction (Chen et al., 2006; Gleason and Im, 2012). We also tend to penalize certain

277 patterns in the configurations in the prior term that tree crowns should not severely overlap.

\subsubsection{Data Term}

279 The data term is in accordance with the aforementioned assumption, indicating the likelihood of the tree 280 objects relative to the low-level segments obtained from the CHM image. Certain geometric features are

281 extracted from the underlying segment of each object, and energy functions are proposed to measure how 282 well those features support the object as a plausible tree.

283 We incorporate the following two energy functions to reflect the assumption: symmetric function $U_{d}^{S}(x)$ 284 and area ratio function $U_{d}^{a}(x)$. The data term is a weighted sum of the two energy functions, subject to a 285 hard constraint on the object radii, according to the following equation: 
This manuscript was accepted for the publication by ISPRS Journal on August 15, 2014. DOI: 10.1016/j.isprsjprs.2014.08.007

$$
U_{d}(\mathbf{x})=\left\{\begin{array}{c}
\sum_{x \in \mathbf{x}}\left(w_{1} U_{d}^{s}(x)+\left(1-w_{1}\right) U_{d}^{a}(x)\right) \text { if } r(x) \in\left[r_{m}, r_{M}\right] \\
+\infty \text { otherwise }
\end{array}\right.
$$

286 where $w_{1}$ is the weight regulating the relative importance of the symmetric and area ratio functions in the 287 data term.

\section{(i) Symmetric Function $U_{d}^{S}(x)$}

289 A symmetric function is defined as a measure of how well a treetop is located in the central part of the 290 crown and the degree to which the tree crown is of a symmetric circular shape. For a given tree object $x$

291 with corresponding segment $s_{x}$, the radii from the treetop point $T$ to the edge of the segment in 8

292 directions with constant angular intervals $\overline{T P_{l}}(i=1, \ldots, 8)$ are first extracted (see Figure 5). The average 293 and standard deviation of the 8 radii are noted as $r(x)$ and $\Delta r(x)$. The asymmetric ratio $R_{\text {sym }}(x) \in[0,1]$

294 of object $x$ is calculated as the coefficient of variance of the radii according to the following equation:

$$
R_{\text {sym }}(x)=\frac{\Delta r(x)}{r(x)}
$$

295 A sigmoid function is then used to define the symmetric function to penalize asymmetric tree crowns

296 given by Eq. (8):

$$
U_{d}^{s}(x)=\frac{1}{1+\exp -\left(\frac{R_{\text {sym }}(x)-\mu_{s}}{\lambda_{s}}\right)}-1
$$

297 where $\mu_{s}$ and $\lambda_{s}$ are parameters set to control the position and slope of the sigmoid function, respectively.

298 The larger the asymmetric ratio $R_{\text {sym }}(x) \in[0,1]$, the higher the symmetric function score $U_{d}^{S}(x) \in$

$299[-1,0]$, which indicates that the treetop is more likely to be a false treetop. 
This manuscript was accepted for the publication by ISPRS Journal on August 15, 2014. DOI: 10.1016/j.isprsjprs.2014.08.007

302 Another area ratio term $U_{d}^{a}(x)$ is included to re-enforce the assessment of the geometric features of the

303 objects in the configuration.

304 Likewise, an area ratio $R_{\text {area }} \in[0,1]$ is first calculated. The ratio is computed as the proportion of the 305 intersection of object $x$ and the underlying segments $s_{x}$ to the entire area of the segments $A\left(s_{x}\right)$ by Eq.

306 (9). As the area ratio increases, the degree of the geometric feature of the object increases, in accordance

307 with the hypothesis (see Figure 6).

$$
R_{\text {area }}(x)=\frac{A\left(x \cap s_{x}\right)}{A\left(s_{x}\right)}
$$

308 Based on the area ratio of the object, the area ratio function is defined according to the following

309 equation:

$$
U_{d}^{a}(x)=\frac{1}{1+\exp -\left(\frac{R_{\text {area }}(x)-\mu_{a}}{-\lambda_{a}}\right)}-1
$$

310 where $\mu_{a}$ and $\lambda_{a}$ are used to control the position and slope of the sigmoid function, respectively.

$311 * * * * *$ Approximate position of Figure $6 * * * * *$

\section{$312 \quad 3.4 .2 \quad$ Prior Term}

313 The prior term introduces a priori knowledge concerning the layout of the objects. In most mature

314 coniferous forest stands, tree crowns will not overlap too severely. However, overlap between objects

315 should not be totally prohibited. A repulsive term is then defined as a soft penalizing function to penalize

316 severe overlaps in the configuration.

\section{7 (i) Overlap Function $\boldsymbol{U}_{\boldsymbol{p}}^{\boldsymbol{o}}(\mathbf{x})$}

318 To define the overlap function, we first introduce a symmetric neighborhood relationship between

319 objects. We say two objects $x_{i}=\left(t_{i}, r_{i}\right)$ and $x_{j}=\left(t_{j}, r_{j}\right)$ are overlapping if the distance between them is 
This manuscript was accepted for the publication by ISPRS Journal on August 15, 2014. DOI: 10.1016/j.isprsjprs.2014.08.007 area of the smaller object, according to the following equation (see Figure 7):

$$
R_{\text {overlap }}\left(x_{i}, x_{j}\right)=\frac{A\left(x_{i} \cap x_{j}\right)}{\min \left(A\left(x_{i}\right), A\left(x_{j}\right)\right)}
$$

323 The overlap score $O\left(x_{i}, x_{j}\right)$ on $x_{i} \sim x_{j}$ is then given according to the following equation:

$$
O\left(x_{i}, x_{j}\right)=\frac{1}{1+\exp -\left(\frac{R_{\text {overlap }}\left(x_{i}, x_{j}\right)-\mu_{o}}{\lambda_{o}}\right)}
$$
where $\mu_{o}$ and $\lambda_{o}$ are set to control the position and slope of the sigmoid function, respectively.

The overlap function of configuration $X$ can be expressed according to the following equation:

$$
U_{p}^{o}(\mathbf{x})=\sum_{x_{i} \sim x_{j}} O\left(x_{i}, x_{j}\right), \forall x_{i}, x_{j} \in \mathbf{x}, i \neq j
$$

*****Approximate position of Figure $7 * * * * *$

327 Compared with a classical Marked Point Process, limiting the search space to configurations generated

328 from a subset of a finite set of seed points $T$ (the pre-extracted local maxima) prevents multiple detection 329 problems. The global energy does not have to be designed to prevent the selection of multiple instances of 330 the same tree because duplicated trees are not part of the search space. Thus, the prior term contains only 331 the overlap function and is written according to the following equation:

$$
U_{p}(\mathbf{x})=U_{p}^{o}(\mathbf{x})
$$

\subsection{Parameter Estimation}

333 Parameters in the model can be distinguished into the following three categories: physical parameters,

334 weights and thresholds. The physical parameters $r_{m}$ and $r_{M}$ are size constraints specifying the range of the 
This manuscript was accepted for the publication by ISPRS Journal on August 15, 2014. DOI: 10.1016/j.isprsjprs.2014.08.007

tree crown radius in the forest plots. These parameters are set as $1.0 \mathrm{~m}$ and $6.0 \mathrm{~m}$, respectively, according to the range of tree sizes in the test sites.

The weights $\alpha$ and $w_{1}$ are assigned to tune the relative importance that we want to grant to different energy terms or functions in the combination (see Eq. (5) and (6)). Both $\alpha$ and $w_{1}$ are set to 0.5 because we place equal importance on those functions in all of our experiments.

To reduce the hand-tuned parameters and to avoid a "trial-and-error" test for parameter setting in most practices, we propose a parameter estimation method to estimate the threshold pair $(\mu, \lambda)$ in the sigmoid functions (Eq. (8), (10), and (12)) in the energy terms. In each function, the threshold pair $(\mu, \lambda)$ controls the tolerance and slope of the sigmoid function, respectively, which plays a significant role in the model. For example, if we set a smaller $\mu_{s}$ value in the symmetric function (Eq. (8)), trees with asymmetric crowns will be penalized more effectively. For a sigmoid function, a smaller value of $\lambda$ results in a steeper slope, and the associated energy function has an increased discriminative behavior of a step function (see

\section{Figure 8).}

*****Approximate position of Figure $8 * * * * *$

We address two issues in the parameter estimation of the energy minimization model. First, the energy terms are designed to penalize false tree objects or implausible configurations with respect to the data term and the prior term. False tree objects or implausible configurations between the objects should receive high energy scores. The parameter estimation is performed by fitting the sigmoid functions to the posterior probability of the features derived from false tree objects or implausible configurations based on the logistic regression model. Second, collection of a large sample size is required to model the posterior probability of those aforementioned features through the Bayesian theorem. In this study, we propose a Monte Carlo-based method, which enables generation of a sufficient number of samples and leads to the estimation of the parameters in the logistic functions. 
This manuscript was accepted for the publication by ISPRS Journal on August 15, 2014. DOI: 10.1016/j.isprsjprs.2014.08.007

For example, we will examine the symmetric function $U_{d}^{S}(\mathbf{x})$. Let us denote the feature, the asymmetric ratio in this case, extracted from a tree object $x_{i}$ as $d$. A random variable $Y=\{0,1\}$ takes the value of 1 if $x_{i}$ is a true tree object or 0 otherwise. Given an observation $d$, the probability that the random variable is derived from a false tree object can be given by the posterior probability, according to the following equation:

$$
p(Y=0 \mid d)=\frac{p(d \mid Y=0) p(Y=0)}{p(d)}
$$

The higher the posterior probability of the object being a false tree, the higher the energy we assign to the object through the energy functions.

According to the Bayesian theorem, the posterior probability can be rewritten as the following:

$$
p(Y=0 \mid d)=\frac{1}{1+L_{i}^{o} P_{i}^{o}}
$$

where $L_{i}^{o}$ is the likelihood ratio, and $P_{i}^{o}$ is the prior ratio, according to the following equations:

$$
\begin{gathered}
L_{i}^{o}=\frac{p(d \mid Y=1)}{p(d \mid Y=0)} \\
P_{i}^{o}=\frac{p(Y=1)}{p(Y=0)}
\end{gathered}
$$

The likelihood ratio $L_{i}^{o}$ can be calculated by modeling the likelihood distributions of features derived from true and false tree objects. A Monte Carlo sampling is utilized to estimate the likelihood distributions. We generate a configurations $\mathbf{x}\left(T_{i}\right)$ from a random subset of local maxima $T_{i} \subset T$, and $\mathbf{x}\left(T_{i}\right)$ is then compared with the reference configuration $\mathbf{x}\left(T^{o}\right)$. Each tree object $x_{j}^{T_{i}}, j=1, \ldots, n\left(T_{i}\right)$ in configuration $\mathbf{x}\left(T_{i}\right)$ is then labeled as true or false. We repeat this process for $n$ ( $n=50$ in our experiments) time, to collect enough samples for features of the true and false tree objects. 
This manuscript was accepted for the publication by ISPRS Journal on August 15, 2014. DOI: 10.1016/j.isprsjprs.2014.08.007

373 The Monte Carlo-based method produces a pool of samples sufficient to model the likelihood

374 distributions of different features. The maximum likelihood method is applied to model the likelihood

375 distributions of the asymmetric ratio, area ratio, and overlap ratio for true and false trees. In practice, we

376 set the prior ratio $P_{i}^{o}$ to 2, which is empirically based on the general detection accuracy achieved by LM-

377 based approaches. The modeled distributions and fitted functions are shown in Figure 9.

*****Approximate position of Figure $9 * * * * *$

\subsection{Model Optimization}

380 In model optimization, we aim to find the configuration of objects that minimizes the global energy $U(\mathbf{x})$

381 in the configuration space $\Omega_{T}$ that we have proposed. This discrete configuration space can be effectively explored using a Markov Chain Monte Carlo sampler coupled with simulated annealing.

An MCMC sampler consists in simulating a discrete Markov chain $\left(X_{t}\right), t \in \mathbb{N}$ on the configuration space

$384 \Omega_{T}$, which converges towards an invariant measure specified by the energy $U(\mathbf{x})$. The sampler performs

385 transitions for one state of the chain to another by proposing a local change of the current configuration.

386 In our application, a configuration of trees $\mathbf{x}\left(T^{k}\right)$ can be solely determined by a subset of local maxima

$387 T^{k} \subset T$ given the CHM image. Once treetops are set as the local maxima $T^{k}$, the tree sizes are decided

388 and directly derived from the corresponding marker-controlled watershed segments. Therefore, finding

389 the optimal configuration of trees $\mathbf{x}\left(T^{*}\right)$ is equivalent to determining the optimal set of local maxima

$390 T^{*} \subset T$. The transition of the chain can be managed by a birth-and-death process in which a local maxima

391 is added to or removed from the current set of local maxima $T^{k}$ to generate a new configuration $\mathbf{x}\left(T^{k+1}\right)$,

392 from a previous configuration $\mathbf{x}\left(T^{k}\right)$. More specifically,

393 - In a birth process, a local maxima $u$ is randomly selected from $T \backslash T^{k}$ and added to the current local maxima set $T^{k}$ to generate a new configuration $\mathbf{x}\left(T^{k+1}\right)$, with $T^{k+1}=T^{k} \cup\{u\}$. 
This manuscript was accepted for the publication by ISPRS Journal on August 15, 2014. DOI: 10.1016/j.isprsjprs.2014.08.007

- In a death process, a local maxima $v$ is randomly selected and removed from the current local maxima set $T^{k}$ to generate a new configuration $\mathbf{x}\left(T^{k+1}\right)$, with $T^{k+1}=T^{k} \backslash\{v\}$.

The move between the configurations is symmetric and accepted with the following probability:

$$
\min \left(1, \exp -\left(U\left(\mathbf{x}\left(T^{k+1}\right)\right)-U(\mathbf{x})\right)\right)
$$

398 Otherwise, the previous set of local maxima is kept: $T^{k+1}=T^{k}$.

399 A simulated annealing is then embedded in the MCMC to find the optimal configuration with the 400 minimum global energy $U(\mathbf{x})$. To perform the simulated annealing, the Gibbs energy $U(\mathbf{x})$ is replaced 401 with $U_{T_{t}}=U(\mathbf{x}) / T_{t} . T_{t}$ is the temperature parameter, which tends toward zero as $t$ approaches $\infty$. A

402 logarithmic decrease ensures the convergence to the global optimum for all of the initial configurations $403 \mathbf{x}_{0}$. In practice, a geometric cooling scheme is preferred to accelerate the process and to give an 404 approximate solution close to the optimal one, for example, use $T_{t}=T_{0} \alpha^{t}$ with $\alpha$ close to 1 , typically $405 \alpha=0.98$.

\subsection{Accuracy Assessment}

To evaluate the performances of the proposed model, the detected trees are compared with the reference data. The comparison results of all of the aggregated trees from the detected trees and the reference data can be classified into the following three categories: the correctly detected trees (correct), trees in the detection results that have no corresponding reference tree (commission) and trees in the reference data

411 not detected (omission). Commission/Omission statistics and the overall detection accuracy are used to 412 quantify the detection results. The calculation of the commission error, omission error and overall 413 accuracy is based on a conventional method of error matrix assessment (Girard, 2003), as shown by Eq. 414 (20)-(22): 
This manuscript was accepted for the publication by ISPRS Journal on August 15, 2014. DOI: 10.1016/j.isprsjprs.2014.08.007

$$
\begin{gathered}
\text { Commission error }=\frac{N_{\text {det }}-N_{\text {cor }}}{N_{\text {det }}} \times 100 \% \\
\text { Omission error }=\frac{N_{\text {ref }}-N_{\text {cor }}}{N_{\text {ref }}} \times 100 \% \\
\text { Overall quality }=\frac{N_{\text {cor }}}{N_{\text {cor }}+\left(N_{\text {det }}-N_{\text {cor }}\right)+\left(N_{\text {ref }}-N_{\text {cor }}\right)} \times 100 \%
\end{gathered}
$$

415 where $N_{\text {cor }}$ is the number of correctly detected trees, $N_{\text {det }}$ is the total number of detected trees by the 416 algorithm, and $N_{\text {ref }}$ is the number of reference trees.

\section{$417 \quad 4 \quad$ Results}

\subsection{Parameter Estimation Results}

Table 1 displays the parameters estimated for the energy functions of the proposed model. We then performed experiments with the estimated parameters on real and simulated forest plots to test the

421 robustness of the model.

422 The parameter $\mu_{s}$ is the threshold in the symmetric function used to penalize tree crowns with high asymmetric ratios. In a forest in which most tree crowns are of regular circular shapes, the value of $\mu_{s}$ can be set relatively smaller to more effectively penalize crowns with asymmetric ratios that exceed this threshold. The threshold $\mu_{a}$ works conversely. Because a larger area ratio indicates a more circular shaped crown, it must be set to a larger value to better penalize tree crowns of a non-circular shape.

427 Parameter $\mu_{o}$ in the overlap function is set to penalize an overlapping situation that exceeds a certain 428 degree, which works similarly to the $\mu_{s}$ parameter. The greater the degree of crown overlap in a forest 429 plot, the larger the $\mu_{o}$ value should be set.

430 The results shown in Table 1 support this reasoning for parameter setting in which the more the tree 431 crowns in the plot are of symmetric circular shape, the smaller the estimated value of $\mu_{s}$, whereas the 432 larger the value of $\mu_{a}$. This reasoning is more explicitly evidenced by the simulated forest plots in which 
This manuscript was accepted for the publication by ISPRS Journal on August 15, 2014. DOI: 10.1016/j.isprsjprs.2014.08.007

the shape irregularity of the tree crowns increases with the increasing degree of canopy overlap from separated to overlapping, which in turn causes an increase in the value of $\mu_{s}$ from 0.32 to 0.45 and the value of $\mu_{o}$ from 0.08 to 0.40 , whereas the value of $\mu_{a}$ decreases correspondingly from 0.82 to 0.72 . This result also confirms the rationality of our proposed method for parameter estimation. We also notice that the smaller the overlap degree of a plot, the smaller the estimated $\lambda$ in the sigmoid function, which indicates a better "threshold" behavior of the associated energy function. This relationship is well in line with the assumption that the simpler the plot situation, the easier the true tree crowns and the false tree crowns can be distinguished.

From the estimation results of the real and simulated forest plots, we also conclude that the degrees of crown overlap of the real forest plots are between the touch and overlap situations in the simulated forest plots. This condition can be observed from the ranges of the estimated values of $\mu_{s}$ and $\mu_{o}$ of the real forest plots, which are between the parameters estimated for the touch and overlap simulated forest plots.

*****Approximate position of Table $1 * * * * *$

\subsection{Detection Results of Real Forest Plots}

We first applied the proposed model with the estimated parameters to the ALS data of the three real forest plots. The detection results of local maxima filtering with a variable window size (also referred to as LM) and the proposed model are illustrated in Figure 10, which shows a good visual assessment of the performances of the two methods.

The LM results are displayed in the first row (Figure 10(a)-(c)). In these images, the red circles with blue crosses in the center represent the corrected detected tree crowns, whereas the green and cyan circles represent the commission and omission errors, respectively. Figure $\mathbf{1 0}$ clearly shows that the LM method is prone to produce commission errors in those coniferous forest plots. This problem is particularly noted in plot 1 and plot 3 in which numerous false treetops occur on the edge of tree crowns because of the branching structure of the pine tree species growing in those plots. Plot 2 is a forest with relatively sparser 
This manuscript was accepted for the publication by ISPRS Journal on August 15, 2014. DOI: 10.1016/j.isprsjprs.2014.08.007

trees, and commission errors primarily occur near the plot boundaries caused by incomplete crown segments and a lack of reference data.

459 The corresponding images in the second row (Figure 10(d)-(f)) show the detection results using the 460 proposed model. As can be easily interpreted, most green circles were successfully removed, indicating 461 that the proposed model could effectively reduce the commission errors. We noticed that a small number of yellow dot line circles appear, which indicate trees over-pruned by the proposed model. From the three images, we can observe that the omission errors produced by the proposed model are primarily trees with small crowns and are severely overlapped by their neighboring larger trees. We also noticed that many commission errors occur at the edge of the plots where crowns are shown incomplete or the reference data are missing.

Table 2 depicts the detailed quantitative assessment of the detection results of the LM and the proposed model. There is an obvious improvement in the results of the proposed model over the LM method on which it is based. The commission errors of the three forest plots significantly decreased, with the largest extend in plot 1 , decreasing from $36.2 \%$ to $10.3 \%$, whereas the omission errors before and after the application of the proposed model remain at similar levels. On average, the overall detection accuracy

472 increased by approximately $15 \%$, comparing results of the proposed model with those of the LM method.

\subsection{Detection Results of Simulated Forest Plots}

476 The proposed model with the estimated parameters applied to the simulated forest plots exhibited similar 477 detection results to those of the real forest plots. The proposed model significantly reduced the 478 commission errors resulting from the LM method in the three simulated forest plots. Figure 11 shows a 479 clear contrast in the detection results of the LM and the proposed model. 
This manuscript was accepted for the publication by ISPRS Journal on August 15, 2014. DOI: 10.1016/j.isprsjprs.2014.08.007

Similarly, by comparing the corresponding images in Figure 11(a)-(c) and Figure 11(d)-(f), it can be observed that nearly all of the green circles (commission errors) in the LM detection results were removed by the proposed model in the three simulated forest plots. Meanwhile, there is only a negligible increase in the number of yellow dot line circles (omission errors). On average, the proposed model increases the overall detection accuracy by approximately 10\% compared with the LM method in all of the cases.

Table 3 gives the exact detection results of the LM method and the proposed model on the three simulated plots. It is interesting to examine the influence of the crown overlap degree on the single tree detection results of the LM method. The overall detection accuracy decreases by approximately $10 \%$ across the three simulated forest plots with an increasing degree of crown overlap from separated to overlapping. This result is primarily because of the increase in the number of omission errors with the increase in the crown overlap. Trees growing by taller trees are more likely to be missed in the LM detection when crowns are more overlapped. However, the commission errors are less affected by the degree of crown overlap, which remains at a similar level for the three forest plots.

$* * * * *$ Approximate position of Figure $11 * * * * *$

*****Approximate position of Table $3 * * * * *$

\subsection{Optimization Process}

Figure 12 presents the statistics associated with the optimization process, using a simulated forest plot with a touching crown as an example. The plots are at the same abscissa scale to simplify the observation of the optimization process. The iteration index is consistently represented on this axis. In all of the experiments, the temperature decrease coefficient $\alpha$ is set to 0.98 , and the temperature is updated every 500 iterations. For a plot with approximately 200 trees, it takes approximately $1.2 \mathrm{e}+5$ iterations for the energy to converge, which is significantly fewer than the total number of configurations $\left(2^{200} \approx 1.6 \mathrm{e}+\right.$ 60 ) in the entire configuration space. The program takes approximately 3 hours to run in Matlab on a processor with a $2.83 \mathrm{GHz}$ frequency. 
This manuscript was accepted for the publication by ISPRS Journal on August 15, 2014. DOI: 10.1016/j.isprsjprs.2014.08.007

504 The first plot (Figure 12(a)) shows the evolution of the temperature in accordance with a geometric

505 cooling scheme, as described in Section 3.6. Figure 12(b) represents the acceptance rate associated with

506 the "birth-and-death" kernel. The move acceptance rates are high at the beginning of the process and tend

507 to progressively decrease and stabilize to 0. Finally, Figure 12(c) plots the global energy. Variations are

508 the highest during the first iterations, and the energy slowly decreases. The decrease becomes faster as the

509 iterations progress and tends to converge slowly to its minimum.

*****Approximate position of Figure $12 * * * * *$

\section{Discussion}

512 In this study, we present a hybrid framework to improve the performance of single tree detection from

513 ALS data by taking advantage of low-level image processing techniques and a high-level probabilistic

514 model. The proposed model is applied on the ALS data of real and simulated coniferous forest plots. The

515 results show the feasibility of our approach, and the detection quality is superior to that obtained by the

516 local maxima filtering based method.

517 The proposed method has been proven to be effective in reduce the commission errors that are introduced

518 by LM in all coniferous forest plots. The LM approach requires a priori knowledge of the relationship

519 between the tree height and the crown size, and the detection accuracy can be significantly influenced by

520 the specification of the relationship. In many cases, this relationship is either hard to obtain or different

521 from study to study because it depends on certain factors, such as tree species, tree age, tree density,

522 crown overlapping, and species composition of the forest plot. Moreover, Falkowski et al. (2006) noted

523 that the relationship between the tree height and the crown size can be weak under certain forest

524 conditions, which is coherent with our case. In this case, when a relationship is designated between the

525 tree height and the crown size, the parameters set for the LM are simply a trade-off between commission

526 and omission errors. We suggested a relative small window size for the LM to over-extract initial

527 'treetops' at the first stage, and the embedded probabilistic model showed its powerfulness in excluding 
This manuscript was accepted for the publication by ISPRS Journal on August 15, 2014. DOI: 10.1016/j.isprsjprs.2014.08.007

528 the false treetops from the final configuration through stochastic inference by considering the spatial 529 layouts and geometric characteristics of the trees in the forest plots.

530 Simulation of forest plots and ALS data provide a valuable tool to examine the performance of tree 531 detection methods under the influence of stem densities and degrees of crown overlap. The detection 532 results evidence the higher the stem density, the more likely the tree crowns are overlapped in the plot, 533 causing smaller trees growing nearby larger trees not easily be detected. The results obtained are coherent 534 with those reported in other studies that denser plots give less accuracy results than sparse plots. The 535 simulated data also provides a fully controlled environment to observe the behavior of the estimated 536 parameters in the designed energy functions with respect to the factor of crown overlap. The increase in 537 crown overlap results in more asymmetric crowns in CHM, which are noted by the estimated parameters 538 and further validate the rationality of the parameter estimation method we proposed. The simulation in 539 our study is intended to test our proposed model under certain key forest variables, i.e., the tree density 540 and crown overlap in our case. Additional sophisticated simulations of forest structure and ALS returns 541 can be found in Morsdorf et al. (2009) and Disney et al. (2010).

542 The detection of single trees from remote sensing data using marked point processes was first performed 543 by Andersen et al. (2002) in an attempt to directly detect trees of a coniferous plot from ALS point clouds 544 using the marked point process in a Bayesian framework. The results have indicated that the algorithm is 545 generally successful in identifying structures associated with individual tree crowns within the forest plot 546 but appears to be sensitive to complex point cloud data. Perrin $(2005,2006)$ has employed marked point 547 processes to detect tree crowns from CIR aerial imageries of plantations, which leads to a continuous 548 search space for the tree objects, in contrast to the proposed method.

549 The stochastic model we proposed is the first to integrate low-level image processing techniques and a 550 high-level probabilistic model into a hybrid framework for single tree detection. The model assembles 551 marked point processes in terms of object modeling and energy formulation. However, in the model, the 
This manuscript was accepted for the publication by ISPRS Journal on August 15, 2014. DOI: 10.1016/j.isprsjprs.2014.08.007

552 parameters of the tree objects are directly derived from low-level representations of LiDAR images

553 produced by traditional image processing techniques rather than random sampling in classical marked

554 point processes. Thus, the model generates a constrained discrete configuration space, in which we

555 sample for the global optimum that contains the final set of detected trees. In this manner, the

556 computation cost is significantly reduced, and the optimization process can be significantly accelerated.

557 The design of proper energy terms is an important issue we attempt to address due to the different types

558 of data we used and the specific manner in which we constructed a configuration. The models used to

559 detect tree crowns in aerial imageries (Perrin et al., 2005, 2006) make use of the distinctive pixel values

560 between the illuminated area near the center of the tree crowns and that of the backgrounds or valleys

561 between the crowns. The contrast between the tree crowns and the background, or treetop areas and

562 valleys between them, can be exaggerated by shadows and stretched spectral or radiometric

563 characteristics in the optical images. However, the elevation differences between those parts in the CHM

564 images are much milder and complex to model than the contrasts in optical imageries. This fact is also the

565 reason we chose a Gibbs energy to measure the morphological characteristics of the tree objects in a

566 configuration, other than a Bayesian framework to model height distributions, considering the complexity

567 required to design a height model valid for all of the trees of various heights and crown forms in the forest

568 area.

569 Parameter estimation is another challenging task in most stochastic models. In this study, we proposed a

570 Monte Carlo-based method to estimate certain key parameters in our model. The Monte Carlo simulation

571 was used to generate random configurations and to create a sufficient number of samples of true and false

572 tree crowns, which enabled the modeling of feature distributions of true and false tree crowns to estimate

573 thresholds in the energy terms. The experimental results on all of the datasets, especially the simulated

574 ones, suggested that the parameter estimation method works reasonably well. 
This manuscript was accepted for the publication by ISPRS Journal on August 15, 2014. DOI: 10.1016/j.isprsjprs.2014.08.007

575 The proposed method has certain inherited drawbacks detecting trees from the rasterized canopy height

576 model, which is incapable of finding suppressed trees under dominant crowns (Hyyppä et al., 2012). The

577 method is designed to detect trees in the dominant layers in the coniferous forest plots of interest.

578 Exploiting 3D information from the ALS point cloud to detect small trees in the lower forest layer is a

579 possible direction to overcome this disadvantage (Ferraz et al., 2012; Reitberger et al., 2009). Another

580 limitation of the method is that it is unable to recover the omission error produced by local maxima

581 filtering on which it is based. Because tree positions are constrained within the pre-extracted local

582 maxima, the model experienced a reduced ability in the classical marked point process to sample the

583 configuration space more thoroughly. However, experimental results on real and simulated forest plots

584 still suggest that the proposed model is a good compromise regarding complexity, efficiency and

585 accuracy.

\section{$586 \quad 6$ Conclusions and Future Studies}

587 We propose a hybrid framework to detect single trees from ALS data by combining the low-level image 588 processing techniques of LM and MCWS with a high-level probabilistic model. More specifically, in this 589 model, tree crowns in an ALS recovered CHM are modeled as objects and are considered as a

590 configuration of circles. The probabilistic model enables the consideration of the geometric characteristics

591 and the pair-wise interactions of objects in the configuration. The LM and MCWS are employed to

592 produce a low-level representation of the image, which provides a constrained configuration space for the

593 probabilistic model to sample for the optimal configuration. We also propose a Monte Carlo-based

594 method to estimate important parameters in the proposed model. The model is proven effective when

595 applied to real and simulated coniferous forest plots. The results show that the proposed model has a

596 distinct improvement in the detection quality over the traditional local maxima filtering based approach

597 by approximately $10 \%$ on all of the datasets. 
This manuscript was accepted for the publication by ISPRS Journal on August 15, 2014. DOI: 10.1016/j.isprsjprs.2014.08.007

598 Future studies should involve a further examination of the optimization methods. An important benefit we

599 gained from our proposed model is that the configuration space is significantly reduced by incorporating

600 features extracted from the CHM image through low-level image processing techniques. However, there

601 remains a significant requirement to accelerate the optimization process. A prior-guided MCMC or a

602 steepest gradient descent algorithm are possibilities we will examine to accelerate the search for the

603 optimal configuration within the discrete configuration space. Second, post-processing will be introduced

604 to recover omission errors from the detection results. Although the proposed model was proven effective

605 in reducing commission errors, the tree positions are constrained in the predetermined set of the local

606 maxima extracted by local maxima filtering. It is possible to recover a portion of the omitted trees from

607 the detected results because those missed crowns will result in more geometrically irregular segments.

608 Finally, automated segmentation of forest stands into homogenous areas with similar forest conditions

609 can be introduced to help train parameters of the proposed model of representative regions and make the

610 model applicable to larger areas. We will also further test the proposed model on more datasets of

611 different forest types and conditions.

\section{Acknowledgements}

613 This research was supported by a grant for a project entitled "Automated Change Detection of 3D

614 Landscape Objects for Powerline Corridor Mapping by Integrating Airborne LiDAR and Multiple-

615 viewing Digital Cameras" funded by Ontario Centres of Excellence and GeoDigitial International Inc. and

616 the Natural Sciences and Engineering Research Council of Canada (NSERC). The authors would like to

617 thank the anonymous reviewers for their valuable comments and suggestions.

\section{References}

619 Andersen, H.-E., Reutebuch, S.E., Schreuder, G.F., 2002. Bayesian object recognition for the analysis of

620 complex forest scenes in airborne laser scanner data, International Archives of Photogrammetry and

621 Remote Sensing, pp. 35-41. 
This manuscript was accepted for the publication by ISPRS Journal on August 15, 2014. DOI: 10.1016/j.isprsjprs.2014.08.007

622 Bortolot, Z.J., Wynne, R.H., 2005. Estimating forest biomass using small footprint LiDAR data: An

623 individual tree-based approach that incorporates training data. ISPRS Journal of Photogrammetry and

624 Remote Sensing 59, 342-360.

625 Brandtberg, T., 2007. Classifying individual tree species under leaf-off and leaf-on conditions using

626 airborne lidar. ISPRS Journal of Photogrammetry and Remote Sensing 61, 325-340.

627 Brandtberg, T., Walter, F., 1998. Automated delineation of individual tree crowns in high spatial

628 resolution aerial images by multiple-scale analysis. Machine Vision and Applications 11, 64-73.

629 Brandtberg, T., Warner, T.A., Landenberger, R.E., McGraw, J.B., 2003. Detection and analysis of

630 individual leaf-off tree crowns in small footprint, high sampling density lidar data from the eastern

631 deciduous forest in North America. Remote Sens. Environ. 85, 290-303.

632 Breidenbach, J., Næsset, E., Lien, V., Gobakken, T., Solberg, S., 2010. Prediction of species specific

633 forest inventory attributes using a nonparametric semi-individual tree crown approach based on fused

634 airborne laser scanning and multispectral data. Remote Sens. Environ. 114, 911-924.

635 Chen, Q., Baldocchi, D., Gong, P., Kelly, M., 2006. Isolating individual trees in a savanna woodland

636 using small footprint lidar data. Photogrammetric Engineering and Remote Sensing 72, 923-932.

637 Descombes, X., Pechersky, E., 2006. Tree Crown Extraction using a Three State Markov Random Field.

638 Descombes, X., Zerubia, J., 2002. Marked point process in image analysis. IEEE Signal Processing

639 Magazine 19, 77-84.

640 Disney, M.I., Kalogirou, V., Lewis, P., Prieto-Blanco, A., Hancock, S., Pfeifer, M., 2010. Simulating the

641 impact of discrete-return lidar system and survey characteristics over young conifer and broadleaf forests.

642 Remote Sens. Environ. 114, 1546-1560.

643 Dubayah, R.O., Drake, J.B., 2000. Lidar Remote Sensing for Forestry Applications. J Forest 98, 44-46.

644 Erikson, M., 2003. Segmentation of individual tree crowns in colour aerial photographs using region

645 growing supported by fuzzy rules. Canadian Journal of Forest Research 33, 1557-1563. 
This manuscript was accepted for the publication by ISPRS Journal on August 15, 2014. DOI: 10.1016/j.isprsjprs.2014.08.007

646 Falkowski, M.J., Smith, A.M.S., Hudak, A.T., Gessler, P.E., Vierling, L.A., Crookston, N.L., 2006.

647 Automated estimation of individual conifer tree height and crown diameter via two-dimensional spatial 648 wavelet analysis of lidar data. Can J Remote Sens 32, 153-161.

649 Ferraz, A., Bretar, F., Jacquemoud, S., Gonçalves, G., Pereira, L., Tomé, M., Soares, P., 2012. 3-D

650 mapping of a multi-layered Mediterranean forest using ALS data. Remote Sens. Environ. 121, 210-223.

651 Gebreslasie, M.T., Ahmed, F.B., van Aardt, J.A.N., Blakeway, F., 2011. Individual tree detection based 652 on variable and fixed window size local maxima filtering applied to IKONOS imagery for even-aged

653 Eucalyptus plantation forests. International Journal of Remote Sensing 32, 4141-4154.

654 Girard, M.C., 2003. Processing of Remote Sensing Data. Taylor \& Francis, London.

655 Gleason, C.J., Im, J., 2012. A Fusion Approach for Tree Crown Delineation from Lidar Data.

656 Photogrammetric Engineering and Remote Sensing 78, 679-692.

657 Gonzalez, R.C., Woods, R.E., 2008. Digital image processing, 3rd ed. Pearson/Prentice Hall, Upper 658 Saddle River, NJ.

659 Gougeon, F.A., 1995. A crown-following approach to the automatic delineation of individual tree crowns 660 in high spatial resolution aerial images. Can J Remote Sens 21, 274-284.

661 Hastings, W.K., 1970. Monte Carlo sampling methods using Markov chains and their applications.

662 Biometrika 57, 97-109.

663 Heinzel, J., Koch, B., 2011. Exploring full-waveform LiDAR parameters for tree species classification.

664 International Journal of Applied Earth Observation and Geoinformation 13, 152-160.

665 Heurich, M., 2008. Automatic recognition and measurement of single trees based on data from airborne

666 laser scanning over the richly structured natural forests of the Bavarian Forest National Park. Forest

667 Ecology and Management 255, 2416-2433.

668 Hyyppä, J., Hyyppä, H., Leckie, D., Gougeon, F., Yu, X., Maltamo, M., 2008. Review of methods of 669 small-footprint airborne laser scanning for extracting forest inventory data in boreal forests. International 670 Journal of Remote Sensing 29, 1339-1366. 
This manuscript was accepted for the publication by ISPRS Journal on August 15, 2014. DOI: 10.1016/j.isprsjprs.2014.08.007

671 Hyyppä, J., Inkinen, M., 1999. Detecting and Estimating Attributes for Single Trees Using Laser Scanner.

672 The Photogrammetric Journal of Finland 16, 27-42.

673 Hyyppä, J., Kelle, O., Lehikoinen, M., Inkinen, M., 2001. A segmentation-based method to retrieve stem

674 volume estimates from 3-D tree height models produced by laser scanners. Geoscience and Remote

675 Sensing, IEEE Transactions on 39, 969-975.

676 Hyyppä, J., Yu, X., Hyyppä, H., Vastaranta, M., Holopainen, M., Kukko, A., Kaartinen, H., Jaakkola, A.,

677 Vaaja, M., Koskinen, J., Alho, P., 2012. Advances in forest inventory using airborne laser scanning.

678 Remote Sensing 4, 1190-1207.

679 Kaartinen, H., Hyyppä, J., Yu, X., Vastaranta, M., Hyyppä, H., Kukko, A., Holopainen, M., Heipke, C.,

680 Hirschmugl, M., Morsdorf, F., Næsset, E., Pitkänen, J., Popescu, S., Solberg, S., Wolf, B.M., Wu, J.C.,

681 2012. An international comparison of individual tree detection and extraction using airborne laser

682 scanning. Remote Sensing 4, 950-974.

683 Korpela, I., Dahlin, B., Schäfer, H., Bruun, E., Haapaniemi, F., Honkasalo, J., Ilvesniemi, S., Kuutti, V.,

684 Linkosalmi, M., Mustonen, J., 2007. Single-tree forest inventory using lidar and aerial images for 3D

685 treetop positioning, species recognition, height and crown width estimation, International Archieves of

686 Photogrammetry and Remote Sensing and spatial information sciences, pp. 227-233.

687 Lacoste, C., Descombes, X., Zerubia, J., 2005. Point processes for unsupervised line network extraction

688 in remote sensing. Pattern Analysis and Machine Intelligence, IEEE Transactions on 27, 1568-1579.

689 Lafarge, F., Descombes, X., Zerubia, J., Pierrot-Deseilligny, M., 2008. Automatic building extraction

690 from DEMs using an object approach and application to the 3D-city modeling. ISPRS Journal of

691 Photogrammetry and Remote Sensing 63, 365-381.

692 Leckie, D., Gougeon, F., Hill, D., Quinn, R., Armstrong, L., Shreenan, R., 2003. Combined high-density

693 lidar and multispectral imagery for individual tree crown analysis. Can J Remote Sens 29, 633-649. 
This manuscript was accepted for the publication by ISPRS Journal on August 15, 2014. DOI: 10.1016/j.isprsjprs.2014.08.007

694 Maltamo, M., Malinen, J., Packaln, P., Suvanto, A., Kangas, J., 2006. Nonparametric estimation of stem 695 volume using airborne laser scanning, aerial photography, and stand-register data. Can J Forest Res 36, $696426-436$.

697 Means, J.E., Acker, S.A., Fitt, B.J., Renslow, M., Emerson, L., Hendrix, C.J., 2000. Predicting forest 698 stand characteristics with airborne scanning lidar. Photogrammetric Engineering and Remote Sensing 66, $699 \quad 1367-1371$.

700 Metropolis, N., Rosenbluth, A.W., Rosenbluth, M.N., Teller, A.H., Teller, E., 1953. Equation of State 701 Calculations by Fast Computing Machines. The Journal of Chemical Physics 21, 1087-1092.

702 Meyer, F., Beucher, S., 1990. Morphological segmentation. Journal of visual communication and image 703 representation 1, 21-46.

704 Morsdorf, F., Nichol, C., Malthus, T., Woodhouse, I.H., 2009. Assessing forest structural and 705 physiological information content of multi-spectral LiDAR waveforms by radiative transfer modelling. 706 Remote Sens. Environ. 113, 2152-2163.

707 Næsset, E., 1997. Determination of mean tree height of forest stands using airborne laser scanner data.

708 ISPRS Journal of Photogrammetry and Remote Sensing 52, 49-56.

709 Naesset, E., 2002. Predicting forest stand characteristics with airborne scanning laser using a practical 710 two-stage procedure and field data. Remote Sens. Environ. 80, 88-99.

711 Naesset, E., 2004. Practical large-scale forest stand inventory using a small-footprint airborne scanning 712 laser. Scand J Forest Res 19, 164-179.

713 Naesset, E., Gobakken, T., Holmgren, J., Hyyppa, H., Hyyppa, J., Maltamo, M., Nilsson, M., Olsson, H., 714 Persson, A., Soderman, U., 2004. Laser scanning of forest resources: The Nordic experience. Scand J 715 Forest Res 19, 482-499.

716 Orka, H.O., Naesset, E., Bollandsas, O.M., 2009. Classifying species of individual trees by intensity and 717 structure features derived from airborne laser scanner data. Remote Sens. Environ. 113, 1163-1174. 
This manuscript was accepted for the publication by ISPRS Journal on August 15, 2014. DOI: 10.1016/j.isprsjprs.2014.08.007

718 Ortner, M., Descombes, X., Zerubia, J., 2008. A marked point process of rectangles and segments for 719 automatic analysis of digital elevation models. Pattern Analysis and Machine Intelligence, IEEE

720 Transactions on 30, 105-119.

721 Perrin, G., Descombes, X., Zerubia, J., 2005. Point processes in forestry: an application to tree crown 722 detection. INRIA.

723 Perrin, G., Descombes, X., Zerubia, J., 2006. A non-Bayesian model for tree crown extraction using 724 marked point processes.

725 Pollock, R., 1996. The automatic recognition of individual trees in aerial images of forests based on a 726 synthetic tree crown image model, Computer Science. University of British Columbia.

727 Popescu, S.C., Wynne, R.H., Nelson, R.F., 2002. Estimating plot-level tree heights with lidar: local 728 filtering with a canopy-height based variable window size. Comput Electron Agr 37, 71-95.

729 Pyysalo, U., Hyyppa, H., 2002. Reconstructing tree crowns from laser scanner data for feature extraction. 730 International Archieves of Photogrammetry and Remote Sensing and spatial information sciences 34, $731 \quad 218-221$.

732 Reitberger, J., Schnörr, C., Krzystek, P., Stilla, U., 2009. 3D segmentation of single trees exploiting full 733 waveform LIDAR data. ISPRS Journal of Photogrammetry and Remote Sensing 64, 561-574.

734 Solberg, S., Naesset, E., Bollandsas, O.M., 2006. Single tree segmentation using airborne laser scanner 735 data in a structurally heterogeneous spruce forest. Photogrammetric Engineering and Remote Sensing 72, $736 \quad 1369-1378$.

737 Sonka, M., Hlavac, V., Boyle, R., 2008. Image processing, analysis, and machine vision, 3rd ed.

738 Thompson Learning, Toronto.

739 Suratno, A., Seielstad, C., Queen, L., 2009. Tree species identification in mixed coniferous forest using 740 airborne laser scanning. ISPRS Journal of Photogrammetry and Remote Sensing 64, 683-693. 
This manuscript was accepted for the publication by ISPRS Journal on August 15, 2014. DOI: 10.1016/j.isprsjprs.2014.08.007

741 Tomppo, E., Nilsson, M., Rosengren, M., Aalto, P., Kennedy, P., 2002. Simultaneous use of Landsat-TM 742 and IRS-1C WiFS data in estimating large area tree stem volume and aboveground biomass. Remote 743 Sens. Environ. 82, 156-171.

744 Tournaire, O., Brédif, M., Boldo, D., Durupt, M., 2010. An efficient stochastic approach for building 745 footprint extraction from digital elevation models. ISPRS Journal of Photogrammetry and Remote 746 Sensing 65, 317-327.

747 Tournaire, O., Paparoditis, N., 2009. A geometric stochastic approach based on marked point processes 748 for road mark detection from high resolution aerial images. ISPRS Journal of Photogrammetry and 749 Remote Sensing 64, 621-631.

750 Van Lieshout, M.N.M., 2000. Markov point process and their applications. Imperial College Press, 751 London.

752 Van Lieshout, M.N.M., 2009. Applications of Stochastic Geometry in Image Analysis, in: Kendall, W.S.,

753 Molchanov, I. (Eds.), New Perspectives in Stochastic Geometry. Oxford University Press, pp. 427-450.

754 Vastaranta, M., Holopainen, M., Yu, X., Haapanen, R., Melkas, T., Hyyppä, J., Hyyppä, H., 2011 a.

755 Individual tree detection and area-based approach in retrieval of forest inventory characteristics from low-

756 pulse airborne laser scanning data. Photogram. J. Fin 22, 1-13.

757 Vastaranta, M., Holopainen, M., Yu, X.W., Hyyppa, J., Makinen, A., Rasinmaki, J., Melkas, T., 758 Kaartinen, H., Hyyppa, H., 2011b. Effects of Individual Tree Detection Error Sources on Forest 759 Management Planning Calculations. Remote Sensing 3, 1614-1626.

760 Vastaranta, M., Kankare, V., Holopainen, M., Yu, X., Hyyppä, J., Hyyppä, H., 2012. Combination of 761 individual tree detection and area-based approach in imputation of forest variables using airborne laser 762 data. ISPRS Journal of Photogrammetry and Remote Sensing 67, 73-79.

763 Vauhkonen, J., Ene, L., Gupta, S., Heinzel, J., Holmgren, J., Pitkanen, J., Solberg, S., Wang, Y.S.,

764 Weinacker, H., Hauglin, K.M., Lien, V., Packalen, P., Gobakken, T., Koch, B., Naesset, E., Tokola, T., 
This manuscript was accepted for the publication by ISPRS Journal on August 15, 2014. DOI: 10.1016/j.isprsjprs.2014.08.007

765 Maltamo, M., 2012. Comparative testing of single-tree detection algorithms under different types of 766 forest. Forestry 85, 27-40.

767 Wang, L., Gong, P., Biging, G.S., 2004. Individual tree-crown delineation and treetop detection in high768 spatial-resolution aerial imagery. Photogrammetric Engineering and Remote Sensing 70, 351-358.

769 White, J., Wulder, M., Vastaranta, M., Coops, N., Pitt, D., Woods, M., 2013. The Utility of Image-Based 770 Point Clouds for Forest Inventory: A Comparison with Airborne Laser Scanning. Forests 4, 518-536.

771 Wulder, M., 1998. Optical remote-sensing techniques for the assessment of forest inventory and 772 biophysical parameters. Progress in physical geography 22, 449-476.

773 Wulder, M., Niemann, K.O., Goodenough, D.G., 2000. Local maximum filtering for the extraction of tree 774 locations and basal area from high spatial resolution imagery. Remote Sens. Environ. 73, 103-114.

775 Xie, Y., Sha, Z., Yu, M., 2008. Remote sensing imagery in vegetation mapping: a review. Journal of Plant 776 Ecology 1, 9-23.

777 Yao, W., Krzystek, P., Heurich, M., 2012. Tree species classification and estimation of stem volume and 778 DBH based on single tree extraction by exploiting airborne full-waveform LiDAR data. Remote Sens.

779 Environ. 123, 368-380.

780 Yu, X.W., Hyyppa, J., Holopainen, M., Vastaranta, M., 2010. Comparison of Area-Based and Individual

781 Tree-Based Methods for Predicting Plot-Level Forest Attributes. Remote Sensing 2, 1481-1495.

782 Yu, X.W., Hyyppa, J., Kaartinen, H., Maltamo, M., 2004. Automatic detection of harvested trees and 783 determination of forest growth using airborne laser scanning. Remote Sens. Environ. 90, 451-462.

784 Zhang, J., Sohn, G., 2010. A comparison of single tree detection algorithm using simulated and real 785 samples, Silvilaser 2010, the 10th International Conference on LiDAR Applications for Assessing Forest 786 Ecosystems, Freiburg, Germany, pp. 480-488. 
This manuscript was accepted for the publication by ISPRS Journal on August 15, 2014. DOI: 10.1016/j.isprsjprs.2014.08.007

787 Table 1: Parameter estimation results of the proposed model for all of the forest plots.

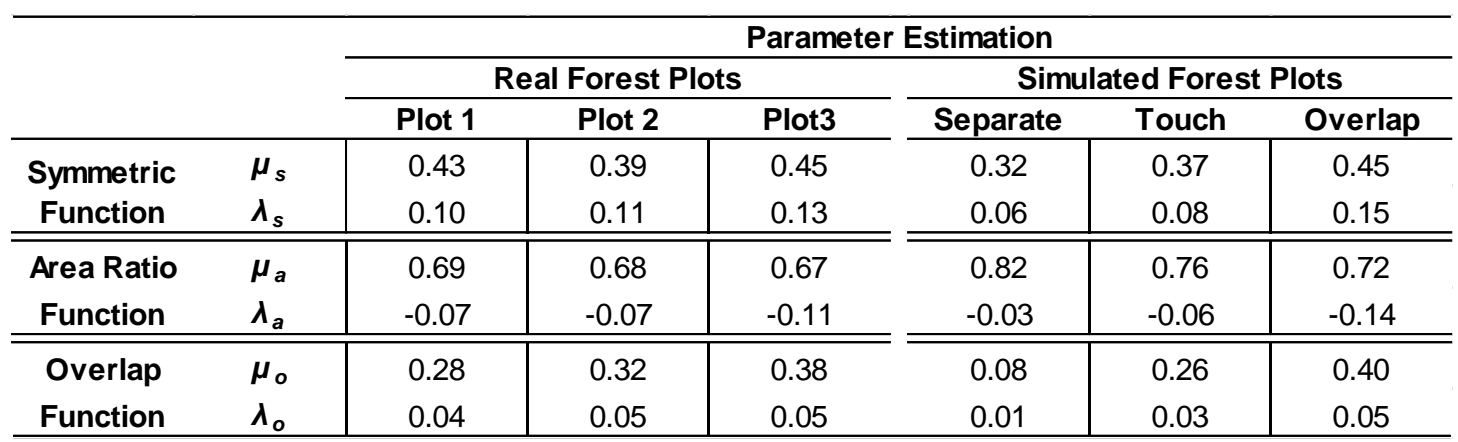

789 Table 2: Detection results of the proposed model with estimated parameters compared with local maxima

790 filtering (LM) on the real coniferous forest plots.

\begin{tabular}{|c|c|c|c|c|c|c|c|c|}
\hline & \multirow{2}{*}{$\begin{array}{l}\text { Detected } \\
\text { Trees }\end{array}$} & \multicolumn{2}{|c|}{ Correct } & \multicolumn{2}{|c|}{ Commission } & \multicolumn{2}{|c|}{ Omission } & \multirow{2}{*}{$\begin{array}{c}\text { Overall } \\
\text { Accuracy }\end{array}$} \\
\hline & & No. & $\%$ & No. & $\%$ & No. & $\%$ & \\
\hline \multicolumn{9}{|l|}{ Plot $1-120$ trees } \\
\hline LM & 185 & 118 & $63.8 \%$ & 67 & $36.2 \%$ & 2 & $1.7 \%$ & $63.1 \%$ \\
\hline Proposed Model & 126 & 113 & $89.7 \%$ & 13 & $10.3 \%$ & 7 & $5.8 \%$ & $85.0 \%$ \\
\hline \multicolumn{9}{|l|}{ Plot 2 - 40 trees } \\
\hline LM & 51 & 38 & $74.5 \%$ & 13 & $25.5 \%$ & 2 & $5.0 \%$ & $71.7 \%$ \\
\hline Proposed Model & 41 & 38 & $92.7 \%$ & 3 & $7.3 \%$ & 2 & $5.0 \%$ & $88.4 \%$ \\
\hline \multicolumn{9}{|l|}{ Plot $3-122$ trees } \\
\hline LM & 141 & 115 & $81.6 \%$ & 26 & $18.4 \%$ & 7 & $5.7 \%$ & $77.7 \%$ \\
\hline Proposed Model & 123 & 112 & $91.1 \%$ & 11 & $8.9 \%$ & 10 & $8.2 \%$ & $84.2 \%$ \\
\hline
\end{tabular}

792 Table 3: Detection results of the proposed model with estimated parameters compared with local maxima

793 filtering (LM) on the simulated forest plots.

\begin{tabular}{|c|c|c|c|c|c|c|c|c|}
\hline & \multirow{2}{*}{$\begin{array}{l}\text { Detected } \\
\text { Trees }\end{array}$} & \multicolumn{2}{|c|}{ Correct } & \multicolumn{2}{|c|}{ Commission } & \multicolumn{2}{|c|}{ Omission } & \multirow{2}{*}{$\begin{array}{c}\text { Overall } \\
\text { Accuracy }\end{array}$} \\
\hline & & No. & $\%$ & No. & $\%$ & No. & $\%$ & \\
\hline \multicolumn{9}{|c|}{ Separate Plot -186 trees } \\
\hline LM & 213 & 184 & $86.4 \%$ & 29 & $13.6 \%$ & 2 & $1.1 \%$ & $85.6 \%$ \\
\hline Proposed Model & 182 & 181 & $99.5 \%$ & 1 & $0.5 \%$ & 5 & $2.7 \%$ & $96.8 \%$ \\
\hline \multicolumn{9}{|c|}{ Touching Plot - 234 trees } \\
\hline LM & 252 & 218 & $86.5 \%$ & 34 & $13.5 \%$ & 16 & $6.8 \%$ & $81.3 \%$ \\
\hline Proposed Model & 216 & 215 & $99.5 \%$ & 1 & $0.5 \%$ & 19 & $8.1 \%$ & $91.5 \%$ \\
\hline \multicolumn{9}{|c|}{ Overlapping Plot - 261 trees } \\
\hline $\mathrm{LM}$ & 256 & 226 & $88.3 \%$ & 30 & $11.7 \%$ & 35 & $13.4 \%$ & $77.6 \%$ \\
\hline Proposed Model & 221 & 221 & $100.0 \%$ & 0 & $0.0 \%$ & 40 & $15.3 \%$ & $84.7 \%$ \\
\hline
\end{tabular}


This manuscript was accepted for the publication by ISPRS Journal on August 15, 2014. DOI: 10.1016/j.isprsjprs.2014.08.007

795 Figure 1: (a) Location of the study area in the Province of Ontario, Canada; (b) a photo and (c) ortho view of the 796 ALS data of a forest plot in the study area rendered by height.

797 Figure 2: (a)-(c) Point process simulated forest plots with different degrees of crown overlap: (a) plot with 798 separated crowns; (b) plot with tree crowns slightly touching each other; (c) plot with overlapping crowns. (d)799 (f) the corresponding ALS point clouds of the three forest plots generated.

$800 \quad$ Figure 3: Flow chart of the proposed method.

801 Figure 4: An example showing the configuration construction from a CHM. (a) a subset of local maxima. Local 802 maxima are shown as red crosses; (b) a marked-controlled watershed segmentation of the CHM using local 803 maxima in (a) as the marker function; (c) the configuration constructed from the local maxima. Radii of the tree crowns are extracted from the corresponding segments in (b).

805 Figure 5: Asymmetric ratio calculation for (a) symmetric and (b) asymmetric tree crowns.

806 Figure 6: Area ratio calculation for tree objects with (a) symmetric and (b) asymmetric tree crowns.

807 Figure 7: Overlap ratio calculation of overlapping tree crowns.

808 Figure 8: Plots of the sigmoid function $F(x)=1 /(1+\exp -(x-\mu) / \lambda)-1$ with respect to different values 809 of $\mu$ and $\lambda$. In the left plot, $\lambda$ is set to 0.2 for all three curves. In the right plot, $\mu$ is set to 0.5 for all three curves.

810 Figure 9: Likelihood distributions, posterior probability and fitted sigmoid functions for the asymmetric ratio, 811 area ratio and overlap ratio. Row 1: Likelihood models of those ratios for the reference group; Row 2: Likelihood 812 models of those ratios for the error group; Row 3: Posterior probabilities (red lines) for those ratios for the error 813 group and the fitted sigmoid functions (blue dashed lines).

814 Figure 10: Detection results of the proposed model with estimated parameters compared with traditional local 815 maxima filtering on real coniferous forest plots. (a)-(c) show the local maxima filtering results; (d)-(f) show the 816 detection result of the proposed model using the corresponding local maxima filtering detection as the initial 817 configuration. (the green circles with triangles in the center represent the commission errors; the cyan dot line 
This manuscript was accepted for the publication by ISPRS Journal on August 15, 2014. DOI: 10.1016/j.isprsjprs.2014.08.007

818 circles represent the omission errors resulting from the LM; the yellow circles represent the omission errors

819 produced by the proposed model.)

820 Figure 11: Detection results of the proposed model with estimated parameters compared with local maxima

821 filtering on simulated forests. (a)-(c) show the local maxima filtering detection on the three simulated forest plots;

822 (d)-(f) show the proposed model detection results using the corresponding local maxima filtering detection as

823 the initial configuration. (the green circles with triangles in the center represent the commission errors; the cyan

824 dot line circles represent the omission errors resulting from the LM; the yellow circles represent the omission

825 errors produced by the proposed model.)

826 Figure 12: Statistics associated with the optimization process of the simulated forest plot with touching crowns:

827 (a) Temperature; (b) Acceptance rate; (c) Global energy. 